Wall

Planungs- und Kontrollsysteme 
Friederike Wall

\section{Planungs- und \\ Kontrollsysteme}

Informationstechnische Perspektiven für das Controlling

Grundlagen - Instrumente - Konzepte

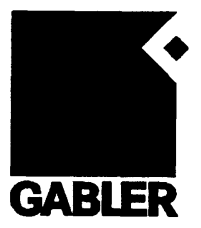


Prof. Dr. Friederike Wall ist Inhaberin des Lehrstuhls für Controlling und Informationsmanagement der Fakultät für Wirtschaftswissenschaft der Universität Witten/ Herdecke.

\section{Die Deutsche Bibliothek - CIP-Einheitsaufnahme}

Wall, Friederike:

Planungs- und Kontrollsysteme : informationstechnische Perspektiven

für das Controlling ; Grundlagen - Instrumente - Konzepte

/ Friederike Wall.

ISBN 978-3-409-13032-5 ISBN 978-3-663-08090-9 (eBook)

DOI 10.1007/978-3-663-08090-9

Alle Rechte vorbehalten

(C) Springer Fachmedien Wiesbaden 1999

Ursprünglich erschienen bei Betriebswirtschaftlicher Verlag Dr. Th. Gabler GmbH, Wiesbaden, 1999

Lektorat: Ulrike Lörcher / Annegret Heckmann

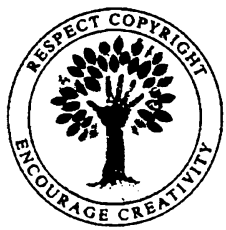

Das Werk einschließlich aller seiner Teile ist urheberrechtlich geschützt. Jede Verwertung außerhalb der engen Grenzen des Urheberrechtsgesetzes ist ohne Zustimmung des Verlages unzulässig und strafbar. Das gilt insbesondere für Vervielfältigungen, Übersetzungen, Mikroverfilmungen und die Einspeicherung und Verarbeitung in elektronischen Systemen.

http://www.gabler-online.de

Höchste inhaltliche und technische Qualität unserer Produkte ist unser Ziel. Bei der Produktion und Verbreitung unserer Bücher wollen wir die Umwelt schonen: Dieses Buch ist deshalb auf säurefreiem und chlorfrei gebleichtem Papier gedruckt. Die Einschweißfolie besteht aus Polyäthylen und damit aus organischen Grundstoffen, die weder bei der Herstellung noch bei der Verbrennung Schadstoffe freisetzen.

Die Wiedergabe von Gebrauchsnamen, Handelsnamen, Warenbezeichnungen usw. in diesem Werk berechtigt auch ohne besondere Kennzeichnung nicht zu der Annahme, daß solche Namen im Sinne der Warenzeichen- und Markenschutz-Gesetzgebung als frei zu betrachten wären und daher von jedermann benutzt werden dürften. 


\section{Vorwort}

Planung und Kontrolle gehören zu den „Klassikern“ der betriebswirtschaftlichen Lehre und Forschung. Angesichts der langen Tradition betriebswirtschaftlicher Auseinandersetzung mit diesem Gebiet muß sich ein neues Lehrbuch mit dem Haupttitel „Planungs- und Kontrollsysteme" der Frage stellen, welche neuen Inhalte, welche neue Herangehensweise oder welche andersartige Perspektive es enthält. Als Ökonom fragt man nach dem zusätzlichen Nutzen dieses neu erstellten „Produktes“. Zur Beantwortung dieser Frage erscheinen mir vor allem zwei aktuelle Anlässe wesentlich:

Den ersten Beweggrund liefert die - nach wie vor kontrovers geführte - Diskussion um die Aufgaben des Controlling. Trotz unterschiedlicher Auffassungen wird die Gestaltung von Planungs- und Kontrollsystemen in der Regel als ein wesentliches Aufgabengebiet des Controlling angesehen. In jüngerer Zeit wird - gerade auf der Basis besonders umfassender Controllingkonzeptionen - die Forderung erhoben, das Planungs- und Kontrollsystem mit anderen führungsrelevanten Teilbereichen der Unternehmung - wie der Organisation - zu koordinieren. $\mathrm{Ob}$ diese Frage noch dem Problembereich des Controlling im Sinne einer eigenständigen Teildisziplin innerhalb der Betriebswirtschaftslehre zugeordnet wird, sei dahingestellt. Wesentlich für die Konzeption dieses Lehrbuchs ist vielmehr, daß eine Koordinationsproblematik mit erheblicher Bedeutung für wirkungsvolle Planungs- und Kontrollsysteme benannt wird. Zwar thematisiert die neuere Controlling-Literatur diesen Koordinationsbedarf zwischen Planungs- und Kontrollsystem einerseits und Organisation auf der anderen Seite, eine nähere Konkretisierung erfährt diese Problematik jedoch kaum. In diesem Buch wird deswegen der Versuch unternommen, den Koordinationsbedarf zwischen Planungs- und Kontrollsystem und Organisation zu präzisieren 'und insbesondere auch auf wesentliche Gestaltungsparameter von Planungs- und Kontrollsystemen zu beziehen.

Den zweiten Anlaß für dieses Buch liefert die moderne Informationstechnik, die - und diese Aussage ist mittlerweile fast eine Selbstverständlichkeit - immer größere Bedeutung in und für Unternehmen erlangt. Dabei wandelt sich die Funktion Informationstechnik-gestützter Systeme zunehmend von „Daten-verarbeitenden Rechen- und Speichermaschinen“ zu eigenständigen Organisationselementen, die die Aufbau- und Prozeßstrukturen eines Unternehmens nicht nur beeinflussen, sondern neu- oder andersartige organisatorische Lösungen (wie z.B. Netzwerkstrukturen) ermöglichen. Diese können mitunter gerade auch deshalb realisiert werden, weil nun bestimmte Planungs- und Kontrollprobleme besser oder überhaupt gelöst werden können: Moderne Decision Support Systeme mit integrierten Methoden- und Modellbanken bieten Planungsträgern individuelle Unterstützung; Groupware-Systeme ermöglichen - geographisch und zeitlich - verteilt ablaufende koordinierte Gruppenentscheidungsprozesse. Diese Entwicklungen versucht das vorliegende Lehrbuch dahingehend aufzunehmen, daß es 
einen Schwerpunkt auf die Beschreibung der erweiterten Gestaltungsmöglichkeiten von Planungs- und Kontrollsystemen im organisatorischen Kontext legt, die durch eine Integration der Potentiale moderner Informationstechnologien heute realisiert werden können. Das Schwergewicht der Darstellung wird dabei auf eine übergreifende Sicht auf Planungs- und Kontrollsysteme gelegt, die neben ,klassischen“ Controllingfragen auch organisatorische und informationstechnische Aspekte einbezieht. An manchen Stellen, z.B. bei der Beschreibung von Planungs- und Kontrollinstrumenten oder der informationstechnischen Infrastrukturen, mußte daher auf detailliertere Darstellungen zugunsten einer breiter angelegten Konzeption verzichtet werden. Der interessierte Leser wird für entsprechende detailliertere Darstellungen auf die einschlägige Literatur der Planungstheorie, des Operations Research sowie der Organisationstheorie oder der Wirtschaftsinformatik verwiesen.

Dieses Lehrbuch wendet sich an Studierende höherer Semester, die sich für die Gestaltung Informationstechnik-gestützter Planungs- und Kontrollsysteme interessieren. Es bietet gleichermaßen auch Praktikern, insbesondere Controllern, die das Planungs- und Kontrollsystem ihres Unternehmens konzipieren, an sich ändernde Rahmenbedingungen anpassen und im laufenden Betrieb steuern, Anregungen.

Ein solches Buch ist kaum ohne Unterstützung zu schreiben. Dieses Buch verdankt viele kritische Anregungen und wertvolle Mithilfe meinen Mitarbeitern Herrn Dipl.-Kfm. Frank Bergsdorf und Herrn Dipl.-Kfm. Bernhard Hirsch. Ihnen möchte ich an dieser Stelle für die hervorragende Zusammenarbeit danken. Dank gebührt auch Frau Gabriela Koerber, die zahlreiche Textabschnitte erfaßt und - mit sehr viel Nachsicht und Geduld - die ständigen Textänderungen in das Manuskript eingearbeitet hat. Bedanken möchte ich mich ferner bei zwei studentischen Mitwirkenden: Herr cand. rer. oec. Thorsten Kertzel hat die zahlreichen Abbildungen mit großer Sorgfalt erstellt. Herr cand. rer. oec Peter Voss hat das Buch auf aus studentischer Sicht Unverständliches gelesen und an den Korrekturarbeiten mitgewirkt. 


\section{Inhaltsverzeichnis}

Abbildungsverzeichnis ................................................................................................

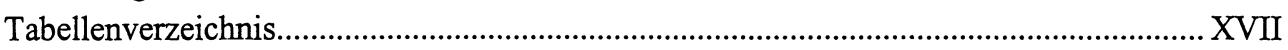

Abkürzungsverzeichnis .................................................................................................

Teil A: Grundlagen .....................................................................................................................................1

1 Zur Konzeption des Buches .............................................................................................

1.1 Interdisziplinarität von Planung und Kontrolle ................................................................

1.2 Informationsorientierung als integrierende Perspektive...................................................

1.3 Zum Aufbau des Buches .......................................................................................

1.4 Wiederholungs- und Vertiefungsfragen ..................................................................

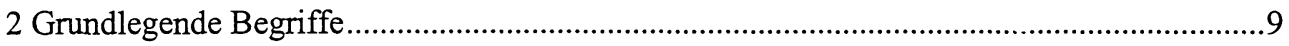

2.1 Planung, Planungssystem und Plansystem.....................................................................

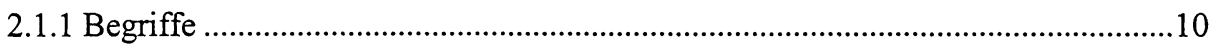

2.1.2 Charakteristische Merkmale.............................................................................12

2.2 Kontrolle, Kontrollsystem und Berichtssystem …………............................................16

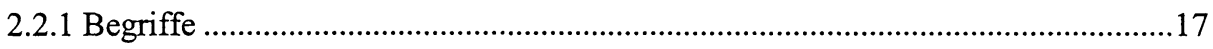

2.2.2 Charakteristische Merkmale.............................................................................19

2.3 Planungs- und Kontrollsystem als Gesamtheit .............................................................21

2.4 Informationen, Informationsbedarf und -bereitstellung, Informationssystem .................25

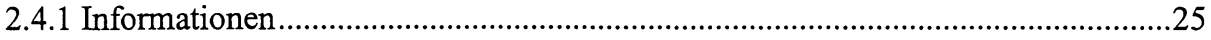

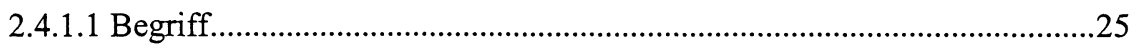

2.4.1.2 Arten und Qualitätseigenschaften.........................................................30

2.4.2 Informationsbedarf und Informationsbereitstellung ……........................................33

2.4.2.1 Begriffe .................................................................................................

2.4.2.2 Prozeß der Informationsbereitstellung........................................................

2.4.2.3 Eigenschaften des Informationsbedarfs ........................................................

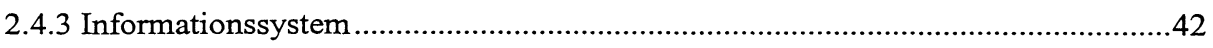

2.5 Wiederholungs- und Vertiefungsfragen .....................................................................

3 Ausgewählte betriebswirtschaftliche Ansatzpunkte für Planungs- und Kontrollsysteme .....45

3.1 Planung und Kontrolle in ausgewählten Theorieansätzen .................................................46

3.1.1 Faktortheoretische Ansatzpunkte .........................................................................

3.1.2 Entscheidungstheoretische Ansatzpunkte ..............................................................49

3.1.3 Systemtheoretische Ansatzpunkte....................................................................5

3.1.4 Institutionenökonomische Ansatzpunkte ...........................................................58 
3.2 Planung und Kontrolle in der Managementlehre ..........................................................60

3.3 Planung und Kontrolle im Controlling.........................................................................63

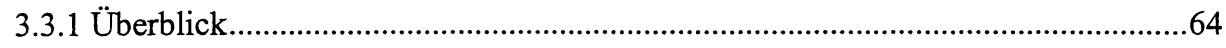

3.3.2 Informationsorientierte Controllingkonzeption .....................................................66

3.3.3 Planungs- und kontrollorientierte Controllingkonzeption ......................................69

3.3.4 Führungssystemorientierte Controllingkonzeption.................................................73

3.3.5 Integrationsansatz für Controllingkonzeptionen ....................................................77

3.4 Wiederholungs- und Vertiefungsfragen ..................................................................

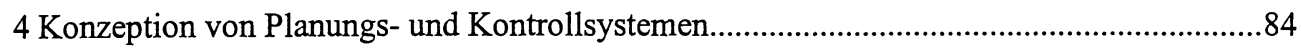

4.1 Differenzierung von Planungs- und Kontrollaufgaben .....................................................85

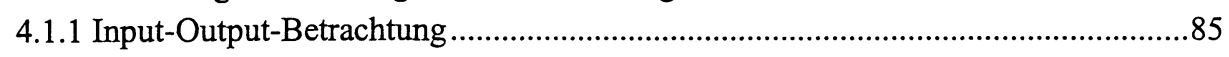

4.1.2 Verrichtungsorientierte Differenzierung .............................................................8

4.1.3 Objektorientierte Differenzierung .......................................................................

4.2 Koordination von Planungs- und Kontrollaufgaben ...................................................93

4.2.1 Funktionaler und instrumentaler Koordinationsbedarf ............................................94

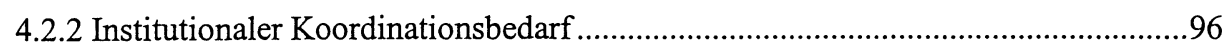

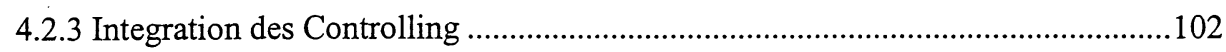

4.2.3.1 Controlling als Koordinationsfunktion.....................................................102

4.2.3.2 Organisatorische Ausgestaltung des Controlling …………………............104

4.3 Integration des Informationsversorgungssystems .......................................................108

4.4 Gesamtsicht der Zusammenhänge...........................................................................111

4.5 Ziele für die Gestaltung von Planungs- und Kontrollsystemen .....................................115

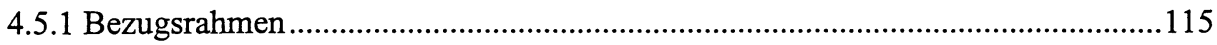

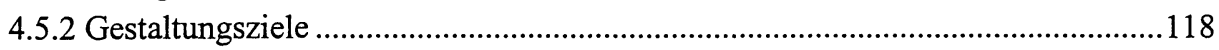

4.5.2.1 Allgemeine Gestaltungsziele ................................................................118

4.5.2.2 Informationsbezogene Gestaltungsziele .................................................121

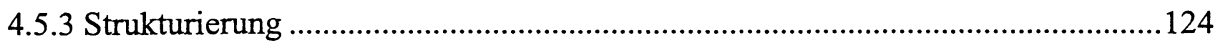

4.5.3.1 Generelle Strukturierungsmöglichkeiten für Ziele ...................................124

4.5.3.2 Zum Aufbau eines Zielsystems für Planungs- und Kontrollsysteme ........126

4.6 Wiederholungs- und Vertiefungsfragen .....................................................................131

Teil B: Instrumente für Planung und Kontrolle .....................................................................133

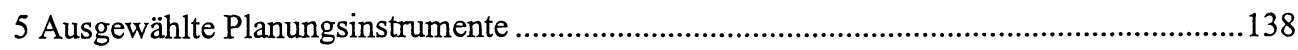

5.1 Instrumente zur Problemerkennung und -analyse .......................................................139

5.1.1 Erfolgsfaktorenanalyse.................................................................................140

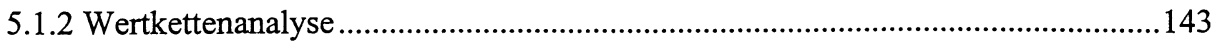

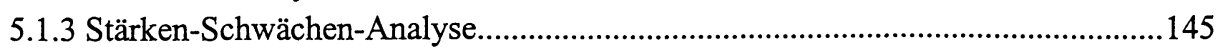




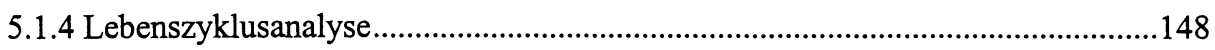

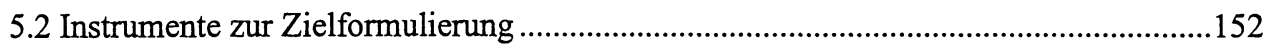

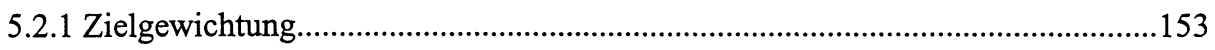

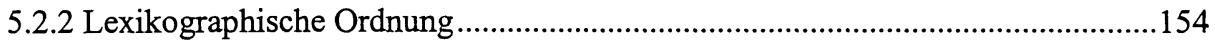

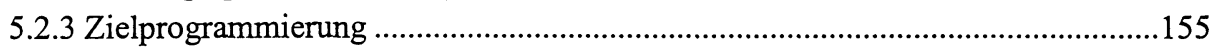

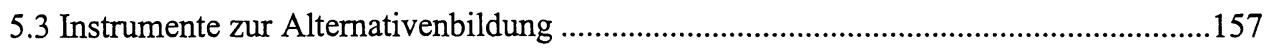

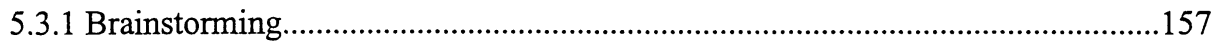

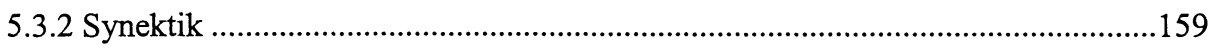

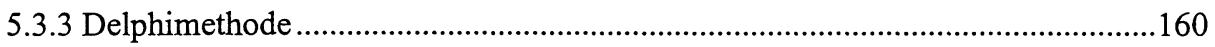

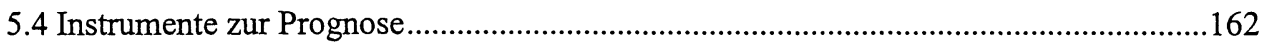

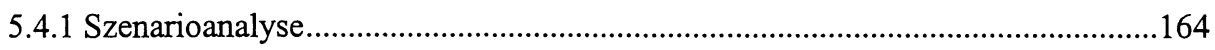

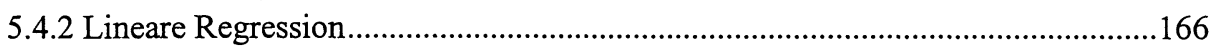

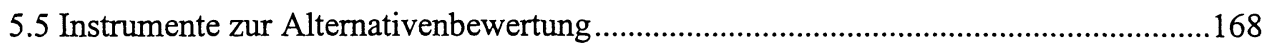

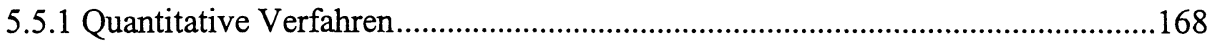

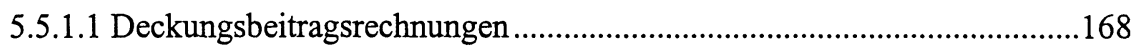

5.5.1.2 Dynamische Verfahren der Investitionsrechnung..................................170

5.5.1.3 Statische Verfahren der Investitionsrechnung ........................................176

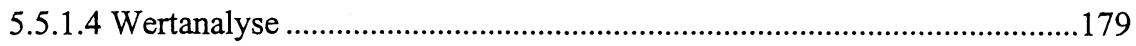

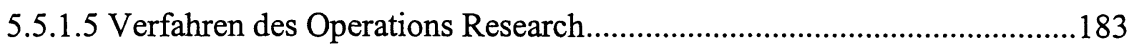

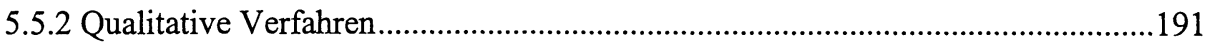

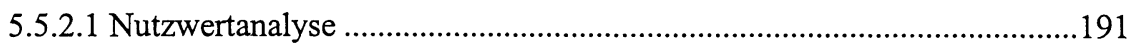

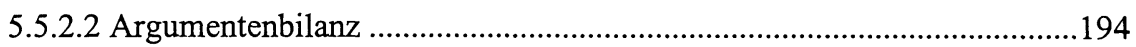

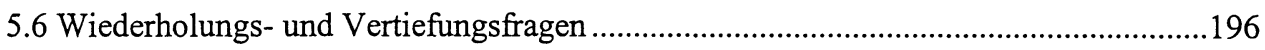

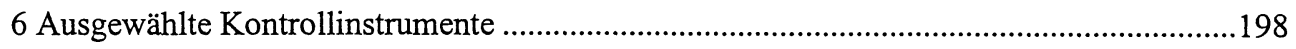

6.1 Instrumente zur Festlegung eines Kontrollbereichs .................................................199

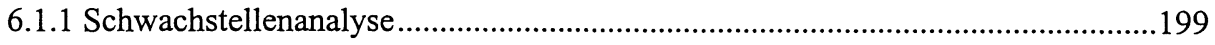

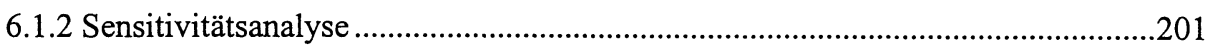

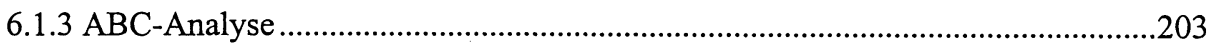

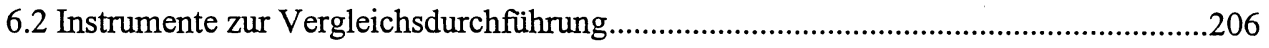

6.2.1 Vergleich mit Hilfe einer starren Plankostenrechnung ......................................207

6.2.2 Vergleich mit Hilfe einer flexiblen Plankostenrechnung auf Vollkostenbasis .....209

6.2.3 Vergleich mit Hilfe einer flexiblen Plankostenrechnung auf Teilkostenbasis......211

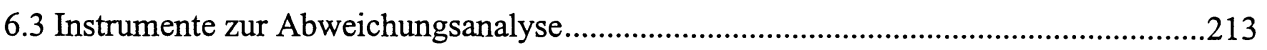

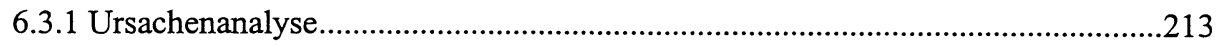

6.3.2 Abweichungsanalyse im Rahmen der Kostenkontrolle .......................................216

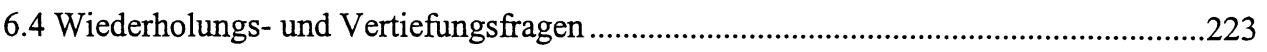


7 Ausgewählte Instrumente für das Planungsmanagement..................................................224

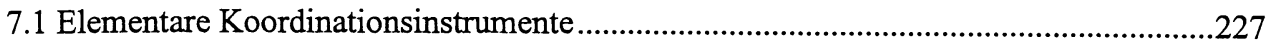

7.1.1 Formen zur inhaltlichen Koordination der Pläne ................................................227

7.1.2 Verfahren der zeitlichen Koordination von Plänen und Berichten ........................231

7.1.3 Verfahren zur Koordination der Planungsreihenfolge .........................................234

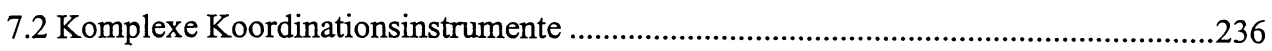

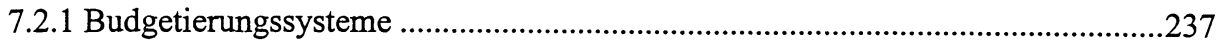

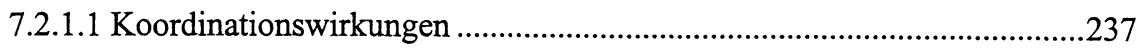

7.2.1.2 Ausgewählte Budgetierungstechniken......................................................241

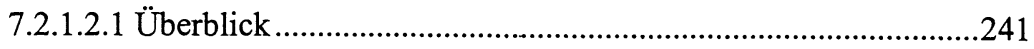

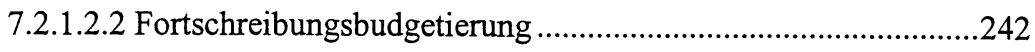

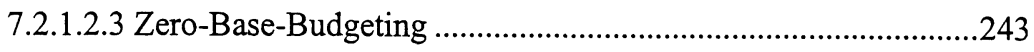

7.2.1.2.4 Zielkostenrechnung (Target Costing)........................................247

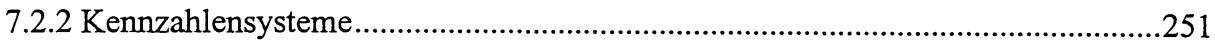

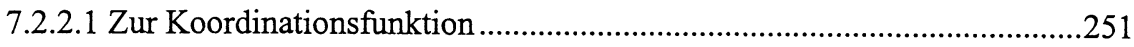

7.2.2.2 Ausgewählte Verfahren zur Entwicklung von Kennzahlensystemen.......255

7.2.2.2.1 Logische Herleitung von Kennzahlensystemen ........................255

7.2.2.2.2 Empirische Fundierung von Kennzahlensystemen ....................257

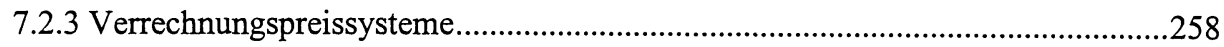

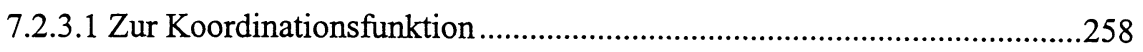

7.2.3.2 Ausgewählte Formen von Verrechnungspreisen ......................................260

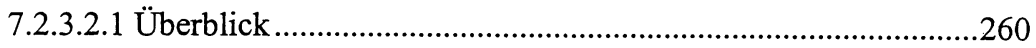

7.2.3.2.2 Marktorientierte Verrechnungspreise ........................................262

7.2.3.2.3 Grenzkostenorientierte Verrechnungspreise ..............................263

7.2.3.2.4 Vollkostenorientierte Verrechnungspreise .................................267

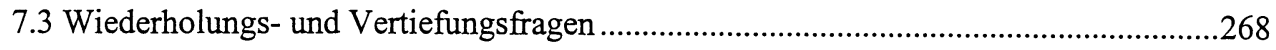

Teil C: IT-gestützte Planungs- und Kontrollsysteme .........................................................271

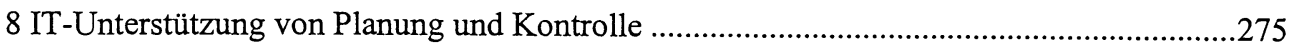

8.1 IT-gestützte PuK-Systeme im Gesamtkonzept IT-basierter Informationssysteme ........276

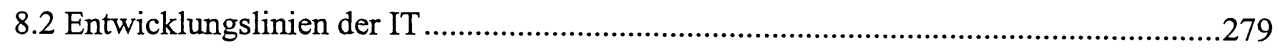

8.2.1 Steigerung der Leistungsfähigkeit und Preisverfall .........................................28

8.2.2 Unterstützung mobiler Arbeitsformen ................................................................282

8.2.3 Unterstützung von Gruppenarbeit (CSCW) ....................................................283

8.2.4 Integration anwendungsbezogener Komponenten ............................................284

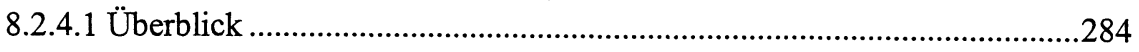

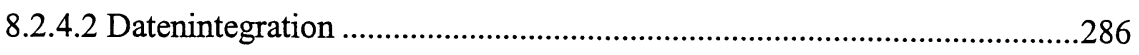

8.2.4.3 Funktions- und Programmintegration.....................................................28

8.2.5 Standardisierung technischer Komponenten..................................................28 
8.2.6 Dezentralisierung von Rechnerkapazitäten, Daten und Programmen...................289

8.2.6.1 Rechnerkapazitäten............................................................................289

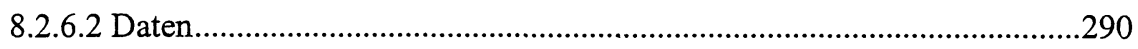

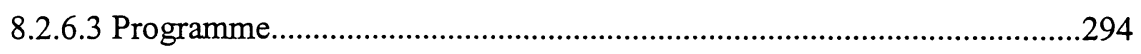

8.3 Spezifische Komponenten und Funktionen IT-gestützter PuK-Systeme ........................294

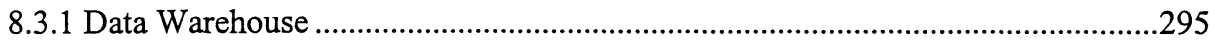

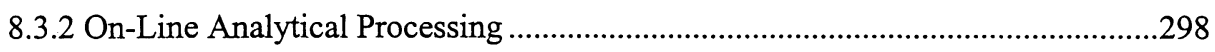

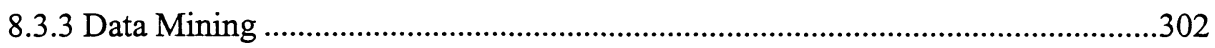

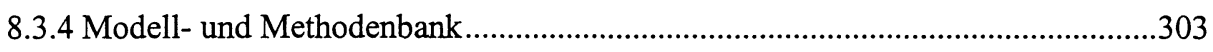

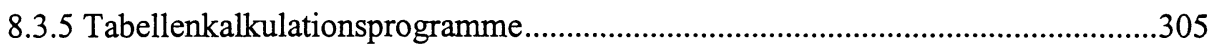

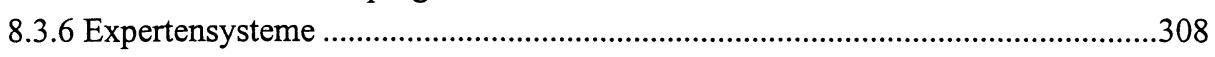

8.4 Spezifische Ausprägungen IT-gestützter Planungs- und Kontrollsysteme .....................311

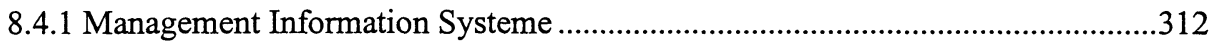

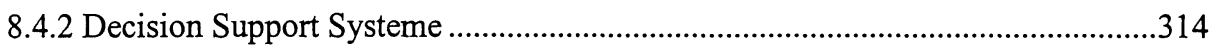

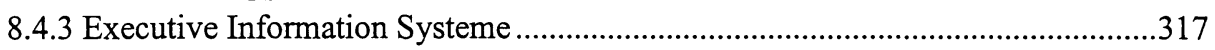

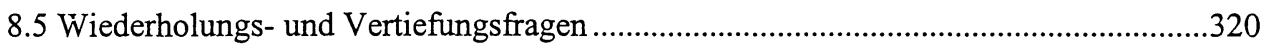

9 Zusammenhänge zwischen Planungs- und Kontrollsystem, Organisationsstruktur und

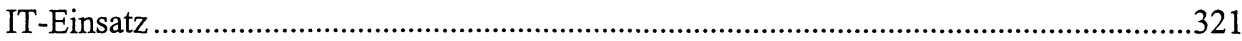

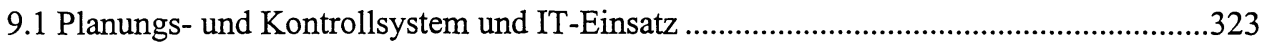

9.1.1 IT-Komponenten als ,Träger“ von Planungs- und Kontrollaufgaben ...................323

9.1.2 Koordinationspotentiale des IT-Einsatzes für Planungs- und Kontrollsysteme ...326

9.1.3 Potentiale des IT-Einsatzes zur Verminderung von Informationsasymmetrien....328

9.2 Planungs- und Kontrollsystem und Organisationsstruktur ..........................................331

9.2.1 Reihenfolge von Planung und Organisation im Führungsprozeß .........................332

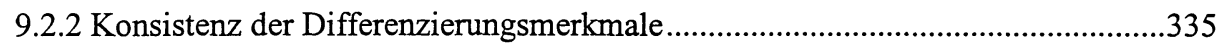

9.2.3 Planungs- und kontrollinduzierte Restriktionen in der Organisationsgestaltung...338

9.2.4 Organisationsstrukturen zur Komplexitäts- und Unsicherheitsreduktion für Planung und Kontrolle.................................................................................341

9.2.5 Planung als organisatorisches Koordinationsinstrument ...................................342

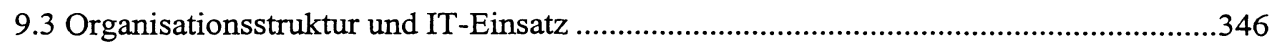

9.3.1 IT-Einsatz als Determinante der Organisationsstruktur (,technological

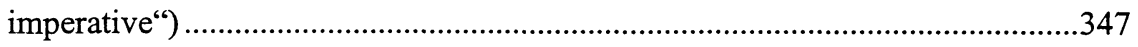

9.3.2 Organisatorische Gestaltungsziele als Determinante des IT-Einsatzes (,organizational imperative“).

9.3.3 IT-bedingte Möglichkeiten in der Organisationsgestaltung (,Enabler“-

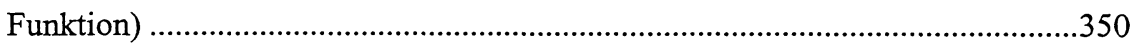

9.3.4 Organisatorische Kompatibilität des IT-Einsatzes („,organizational fit" ${ }^{\circ}$ ) ..............352 


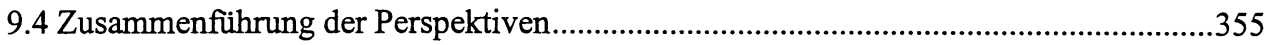

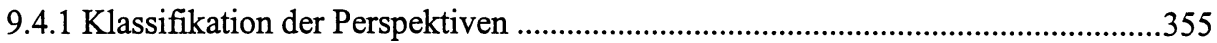

9.4.2 $\mathrm{Zu}$ Wechselwirkungen und Isolierbarkeit von Organisationsgestaltung und

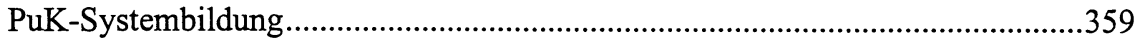

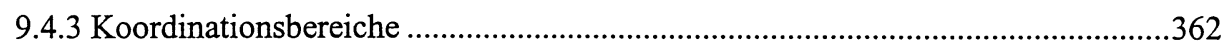

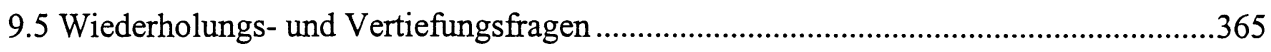

10 Grundstruktur IT-gestützter Planungs- und Kontrollsysteme in ausgewählten

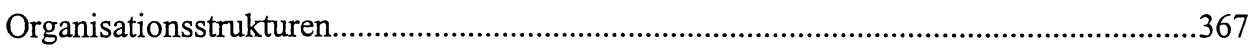

10.1 Planungs- und Kontrollsysteme in funktionalen Organisationen..................................367

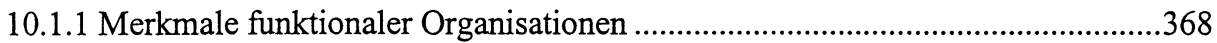

10.1.2 Differenzierung von Planungs- und Kontrollaufgaben ......................................369

10.1.3 Zuordnung auf Planungs- und Kontrollträger ......................................................373

10.1.4 IT-Unterstützung für Planung und Kontrolle ...................................................378

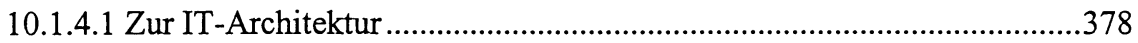

10.1.4.2 Ausgewählte funktionsbezogene Berichtsstrukturen...............................382

10.2 Planungs- und Kontrollsysteme für divisionale Organisationen ....................................388

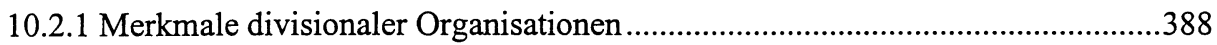

10.2.2 Differenzierung von Planungs- und Kontrollaufgaben .......................................390

10.2.3 Zuordnung auf Planungs- und Kontrollträger ......................................................395

10.2.3.1 Zuordnung in der dezentralen Divisionalorganisation .............................395

10.2.3.2 Zuordnung in der zentralen Divisionalorganisation ...............................397

10.2.4 IT-Unterstützung für Planung und Kontrolle .................................................400

10.3 Planungs- und Kontrollsysteme für netzwerkartige Organisationsstrukturen?.............405

10.3.1 Ausprägungsformen von Netzwerkstrukturen …………....................................406

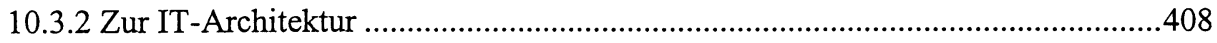

10.3.3 Netzwerkweite Planung und Kontrolle? .............................................................410

10.3.4 Mögliche Planungs- und Kontrollträger …………….......................................415

10.4 Wiederholungs- und Vertiefungsfragen .....................................................................420

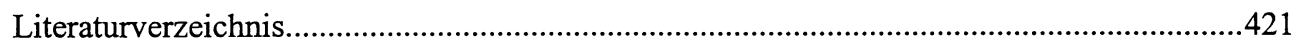

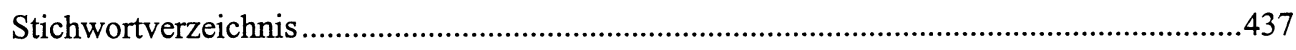




\section{Abbildungsverzeichnis}

Abb. A-1: Begriffe in Zusammenhang mit betriebswirtschaftlicher Planung........................12

Abb. A-2: Begriffe in Zusammenhang mit betriebswirtschaftlicher Kontrolle......................19

Abb. A-3: Planung und Kontrolle als Regelkreis nach Pfohl..............................................24

Abb. A-4: Typologie von Informationsbegriffen nach Bode .................................................27

Abb. A-5: Abgrenzung des Informationsbegriffs für die weiteren Ausführungen

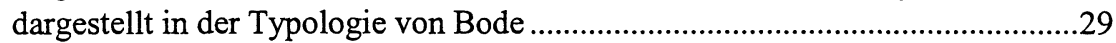

Abb. A-6: Zusammenhang zwischen Informationsbedarf, -nachfrage und -angebot.............34

Abb. A-7: Zusammenhänge zwischen dem potentiellen, objektiven und subjektiven

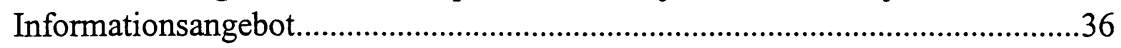

Abb. A-8: Abgrenzungen des Informationssystems...........................................................

Abb. A-9: Phasenschema des Entscheidungsprozesses nach Heinen......................................53

Abb. A-10: Die sechs Schritte des ganzheitlichen Problemlösungsansatzes ..........................61

Abb. A-11: Klassifikation von Controllingkonzeptionen in Anlehnung an Schweitzer/Friedl

Abb. A-12: Informationsorientierte Controllingkonzeption nach Reichmann .........................68

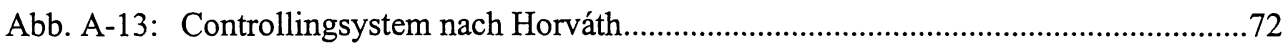

Abb. A-14: Controlling im Führungssystem der Unternehmung nach Küpper .......................76

Abb. A-15: Idealtypischer Führungsprozeß nach Weber ........................................................

Abb. A-16: Einfache Input-Output-Betrachtung für Informationsverarbeitungsprozesse .......86

Abb. A-17: Einfache Input-Output-Betrachtung für Planung und Kontrolle..........................87

Abb. A-18: Input-Output-Betrachtung für einen idealtypischen Planungsprozeß ...................90

Abb. A-19: Input-Output-Betrachtung für den idealtypischen Kontrollprozeß ......................91

Abb. A-20: Beispiel für ein Plansystem ...........................................................................93

Abb. A-21: Zusammenhänge zwischen funktionaler und institutionaler Sicht des Planungssystems

Abb. A-22: Zuordnung von Verfahren und Sachmitteln im Planungssystem........................100

Abb. A-23: Eingliederungsmöglichkeiten des Controlling in die

Unternehmenshierarchie.

Abb. A-24: Gesamtmodell der Zusammenhänge zwischen Planungs- und Kontrollsystem, Informationsversorgungssystem und Controlling

Abb. A-25: Ziel-Mittel-Beziehungen zwischen Unternehmenszielen, Planungs- und Kontrollsystem und Controlling...

Abb. A-26: Exemplarische Gliederungsansätze für Ziele 
Abb. B-1: Instrumente zur Bildung von PuK-Systemen....................................................136

Abb. B-2: Instrumente für den laufenden Einsatz von PuK-Systemen ................................138

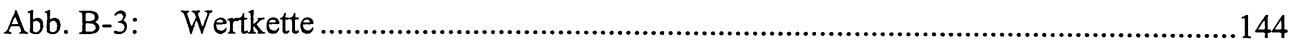

Abb. B-4: Beispiel einer Stärken-Schwächen-Analyse......................................................146

Abb. B-5: Beispiel eines Marktanteil-Marktwachstum-Portfolios ......................................147

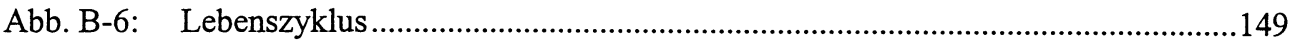

Abb. B-7: Klassifikation von Prognosemethoden .............................................................163

Abb. B-8: Grundmodell der Szenario-Technik .............................................................164

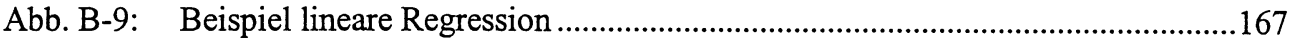

Abb. B-10: Klassische Verfahren der Investitionsrechnung ...............................................171

Abb. B-11: Amortisationsdauer der Zahlungsreihe aus Tab. B-7 ........................................173

Abb. B-12: Interne Zinsfüße bei unterschiedlichen Kapitalwertfunktionen ..........................174

Abb. B-13: Modifizierte Interne-Zinsfuß-Methode nach Baldwin ......................................175

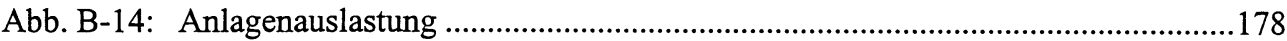

Abb. B-15: Struktur des Wertanalysearbeitsplans nach DIN 69910 ……............................180

Abb. B-16: Zuordnung von Funktionen und Kosten im Rahmen der Wertanalyse ................181

Abb. B-17: Zuordnung von Funktionen und Kosten am Beispiel des Wertanalyse-

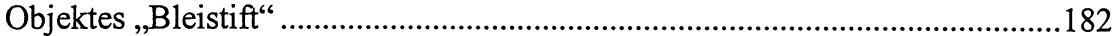

Abb. B-18: Produktionsprogrammplanung mit einem Tabellenkalkulationsprogramm.......187

Abb. B-19: Netzplandarstellung eines Projektes.................................................................190

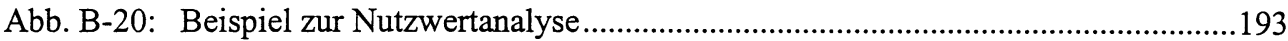

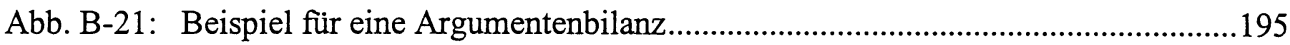

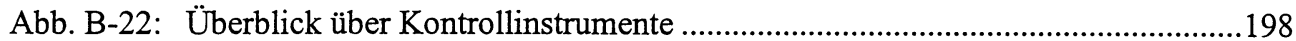

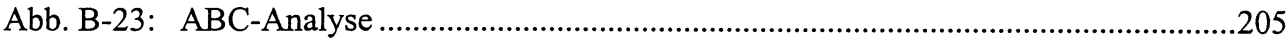

Abb. B-24: Starre Plankostenrechnung ..........................................................................208

Abb. B-25: Flexible Plankostenrechnung auf Vollkostenbasis.............................................211

Abb. B-26: Flexible Plankostenrechnung auf Teilkostenbasis .............................................212

Abb. B-27: Ursachen von Kostenabweichungen .............................................................215

Abb. B-28: Preis- und Mengenabweichungen ersten und zweiten Grades .............................218

Abb. B-29: Kompensierende Abweichungen ersten und zweiten Grades ............................219

Abb. B-30: Überblick über Instrumente für das Planungsmanagement im Rahmen der systemkoppelnden Koordination.....................................................................225

Abb. B-31: Zeitliche Abstimmung von Plänen durch Reihung, Staffelung und

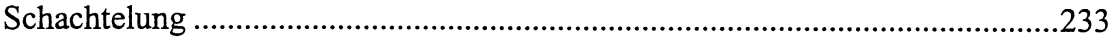

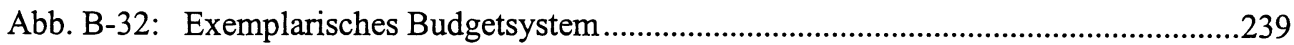

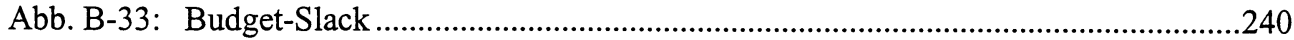

Abb. B-34: Überblick über Budgetierungstechniken .........................................................242

Abb. B-35: Verfahrensschritte des Zero-Base-Budgeting ...................................................244 
Abb. B-36: Abteilungsbezogene Entscheidungspakete und abteilungsübergreifende Rangordnung beim Zero-Base-Budgeting .246

Abb. B-37: Teilschritte der Zielkostenrechnung ............................................................248

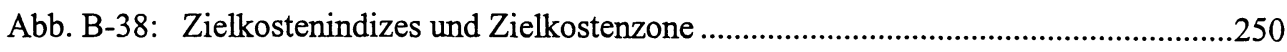

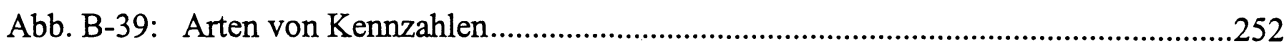

Abb. B-40: Du-Pont-Kennzahlensystem.................................................................256

Abb. B-41： Formen von Verrechnungspreisen................................................................261

Abb. C-1: Gesamtkonzeption betrieblicher Anwendungssysteme nach Mertens ...............277

Abb. C-2: Begriffsabgrenzungen zu IT-gestützten Planungs- und Kontrollsystemen.........279

Abb. C-3: Kapazitätssteigerung und Preisverfall im Hardware-Bereich .............................281

Abb. C-4: Systematisierung der Integrationsformen im Rahmen IT-gestützter

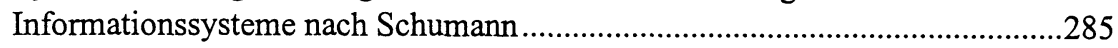

Abb. C-5: Architektur eines Datenbanksystems .........................................................291

Abb. C-6: Prinzipskizze eines physisch verteilten Konzepts ..................................................293

Abb. C-7: Grobarchitektur des Data Warehouse-Konzeptes .............................................297

Abb. C-8: Hyperwürfel in einem OLAP-System und mögliche Datenschnitte ....................300

Abb. C-9: Grobarchitektur des OLAP-Konzeptes ............................................................301

Abb. C-10: Computergestützte Planung mit Hilfe von Modell- und Methodenbanken ........305

Abb. C-11: Architektur eines Tabellenkalkulationsprogramms.............................................306

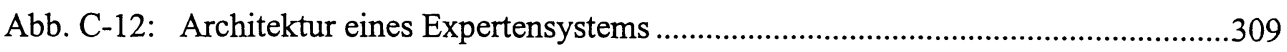

Abb. C-13: Aufbau der Darstellung zu Beziehungen zwischen PuK-System, Organisationsstruktur und IT-Einsatz

Abb. C-14: Beispiel für die Nutzeffektkette bei der Einführung von Scannerkassen.............325

Abb. C-15: Kommunikationsbeziehungen bei zentraler Unternehmensplanung durch einen Planungsstab

Abb. C-16: Beispiel 1 zu Wirkungsfolgen: IT-induzierte Änderungen von PuK-System und Organisationsstruktur

Abb. C-17: Beispiel 2 zu Wirkungsfolgen: Planungsinduzierte Änderungen von Organisationsstruktur und IT-Einsatz ....

Abb. C-18: Beispiel 3 zu Wirkungsfolgen: Strategieinduzierte Änderungen von Organisationsstruktur, Planungssystem und IT-Einsatz.

Abb. C-19: Grundschema eines integrierten Gesamtplanungssystems nach Hahn................370

Abb. C-20: Gesamtplanungssystem für eine Funktionalorganisation nach Hahn...................372

Abb. C-21: Prinzipskizze der Planungsträger in einer Funktionalorganisation .....................377

Abb. C-22: Beispiel einer produktorientierten Divisionalorganisation mit heterogenem Produktprogramm der Produktbereiche

Abb. C-23: Beispiel einer regionalorientierten Divisionalorganisation mit homogenem Produktprogramm der Regionalbereiche. 
Abb. C-24: Grundstruktur eines integrierten Planungssystems für eine Divisionalorganisation nach Hahn ....

Abb. C-25: Prinzipskizze der Zuordnung von Planungsaufgaben auf Planungsträger in einer dezentralen Divisionalorganisation.

Abb. C-26: Prinzipskizze der Zuordnung von Planungsaufgaben auf Planungsträger in einer zentralen Divisionalorganisation

Abb. C-27: Koordinationsbeziehungen in einer Netzwerkorganisation

Abb. C-28: Prinzipskizze der Zuordnung von Planungsaufgaben in einer Netzwerkstruktur mit zentraler Koordinationsstelle 


\section{Tabellenverzeichnis}

Tab. A-1: Teilaktivitäten der Planung …………………….............................................16

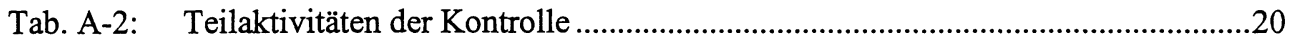

Tab. A-3: Überblick über ausgewählte Controllingkonzeptionen ..........................................78

Tab. A-4: Inhaltliche Bestandteile eines Plans nach Wild.....................................................8

Tab. A-5: Argumente für und gegen Controllingeinheiten................................................104

Tab. A-6: Anforderungen an Planungs- und Kontrollsysteme ...........................................128

Tab. A-7: Ziele für Planungs- und Kontrollsysteme in Übertragung der Zielklassifikation von Hamel ..............................................................................129

Tab. B-1: Einsatzmöglichkeiten von Instrumentengruppen in den Planungsphasen .........139

Tab. B-2: Gegenüberstellung Kreativitätsprozeß - Synektik ..............................................159

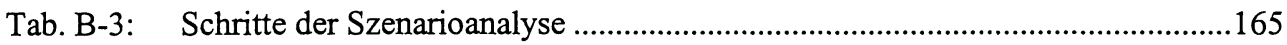

Tab. B-4: Beispieldaten zur Regressionsanalyse.........................................................166

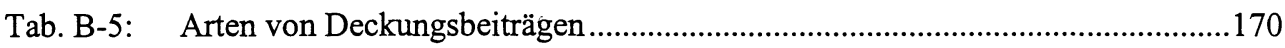

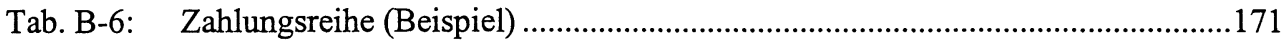

Tab. B-7: Beispieldaten für die Berechnung der Amortisationsdauer................................173

Tab. B-8: Beispiel Produktionsplanung: Benötigte Kapazitätseinheiten und

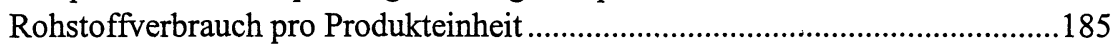

Tab. B-9: Beispiel für die alternative Abweichungsanalyse ..............................................220

Tab. B-10: Beispiel für die einfach-kumulative Abweichungsanalyse ................................221

Tab. B-11: Änderung der Reihenfolge der Abweichungsermittlung beim einfach-

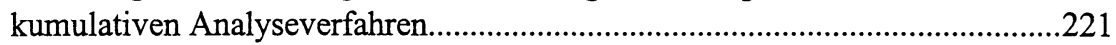

Tab. B-12: Beispiel für die differenziert-kumulative Abweichungsanalyse ........................222

Tab. B-13: Beispielhafte Differenzierungsmerkmale der Unternehmensplanung in Teilplanungen.

Tab. B-14: Merkmale von strategischer, taktischer und operativer Planung nach Küpper

Tab. B-15: Inhaltliche Koordinationsformen der Planung ..............................................230

Tab. B-16: Zeitliche Planungsmerkmale und Koordinationsformen in Anlehnung an Gaitanides.

Tab. B-17: Beispiel zu grenzkostenorientierten Verrechnungspreisen bei linearen Kostenfunktionen bei dezentraler Entscheidung

Tab. B-18: Beispiel zu grenzkostenorientierten Verrechnungspreisen bei linearen Kostenfunktionen bei zentraler Entscheidung..

Tab. B-19: Beispiel zu grenzkostenorientierten Verrechnungspreisen bei nicht-linearen

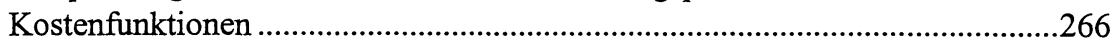

Tab. C-1: Systematisierung von Formen computerunterstützter Gruppenarbeit................283

Tab. C-2: Merkmale typischer MIS nach Gluchowski/Gabriel/Chamoni 
Tab. C-3: Merkmale typischer DSS nach Gluchowski/Gabriel/Chamoni...........................316

Tab. C-4: Merkmale typischer EIS nach Gluchowski/Gabriel/Chamoni .............................319

Tab. C-5: Überblick über die beschriebenen Beziehungen zwischen PuK-System, ITEinsatz und Organisationsstruktur .......................................................................357

Tab. C-6: Auswirkungen auf „Gestaltungsvariable“ von Planungs- und Kontrollsystem, Organisationsstruktur und IT-Einsatz.........................................358

Tab. C-7: Überblick über Berichtsinformationen im Absatzbereich....................................384

Tab. C-8: Überblick über Berichtsinformationen im Produktionsbereich............................386 


\section{Abkürzungsverzeichnis}

\begin{tabular}{|c|c|}
\hline Abb. & Abbildung \\
\hline Anm. & Anmerkung \\
\hline $\mathrm{AOS}$ & Accounting, Organizations and Society \\
\hline ASQ & Administrative Science Quarterly \\
\hline Aufl. & Auflage \\
\hline $\mathrm{Bd}$. & Band \\
\hline $\mathrm{BDE}$ & Betriebsdatenerfassung \\
\hline $\mathrm{BFuP}$ & Betriebswirtschaftliche Forschung und Praxis \\
\hline bzw. & beziehungsweise \\
\hline CACM & Communications of the Association for Computing Machinery \\
\hline CAQ & Computer Aided Quality Assurance \\
\hline CSCW & Computer Supported Cooperative Work \\
\hline d.h. & das heißt \\
\hline DB & Deckungsbeitrag \\
\hline DBMS & Data Base Management System \\
\hline DBW & Die Betriebswirtschaft \\
\hline $\mathrm{DDL}$ & Data Description Language \\
\hline Diss. & Dissertation \\
\hline DML & Data Manipulation Language \\
\hline DNC & Digital Numerical Control \\
\hline DSDL & Data Storage Description Language \\
\hline DSS & Decision Support System \\
\hline DV & Datenverarbeitung \\
\hline DW & Data Warehouse \\
\hline ebd. & ebenda \\
\hline EDI & Electronic Data Interchange \\
\hline EDV & Elektronische Datenverarbeitung \\
\hline EIS & Executive Information System \\
\hline E-mail & electronic mail \\
\hline etc. & et cetera \\
\hline EUS & Entscheidungsunterstützungssystem \\
\hline FIS & Führungsinformationssystem \\
\hline $\mathrm{FuE}$ & Forschung und Entwicklung \\
\hline f. & folgende \\
\hline ff. & fortfolgende \\
\hline GDSS & Group Decision Support System \\
\hline
\end{tabular}


GE Geldeinheiten

ggf. gegebenenfalls

HBR Harvard Business Review

HMD Handbuch der modernen Datenverarbeitung

Hrsg. Herausgeber

i.d.R. in der Regel

i.e.S. im engeren/eigentlichen Sinne

i.S.v. im Sinne von

i.w.S. im weiteren Sinne

insbes. insbesondere

IT Informationstechnologie

IV Informationsverarbeitung

JCL Job Control Language

Jg. Jahrgang

krp Kostenrechnungspraxis

LE Leistungseinheiten

log. logarithmisch

Mbit Megabit

Mbyte Megabyte

MIPS Millonen Instruktionen pro Sekunde

MIS Management Information System

MP Maßnahmenplanung

MSS Management Support System

o.V. ohne Verfasser

OLAP On-Line Analytical Processing

OR Operations Research

OS Organisationsstruktur

p.a. per annum

PC Personal Computer

PCL Process Control Language

PIMS Profit Impact of Market Strategies

PPS Produktionsprogrammplanung und -steuerung

PS Planungssystem

PuK Planung(s-) und Kontroll(e)

QL Query Language

ROLAP Relational On-Line Analytical Processing

S. Seite

s. siehe

SOFT Strenghts, Opportunities, Failures and Threats 
sog. sogenannte(r)

$\mathrm{SzU} \quad$ Schriften zur Unternehmensführung

Tab. Tabelle

TCP/IP Transmission Control Protocol/ Internet Protocol

u.a. und andere

u.E. unseres Erachtens

US United States

v.a. vor allem

VDI Verein Deutscher Ingeneure

Verf. Verfasser(s)

Vol. Volume

VP Verrechnungspreis

WBS Wissensbasierte Systeme

WI Wirtschaftsinformatik

WiSt Wirtschaftswissenschaftliches Studium

WISU Das Wirtschaftsstudium

XPS Expertensystem

z.B. zum Beispiel

ZBB Zero Base Budgeting

ZDE Zeitdatenerfassung

ZfB Zeitschrift für Betriebswirtschaft

$\mathrm{ZfbF} \quad$ Zeitschrift für betriebswirtschaftliche Forschung

ZFO Zeitschrift Führung und Organisation

ZfO Zeitschrift für Organisation

ZP Zeitschrift für Planung 